\title{
Energy economics and climate policy modeling
}

\author{
Zhimin Huang1,2 . Yi-Ming Wei ${ }^{2,3,4,5}$. \\ Ke Wang $2,3,4,5$. Hua Liao ${ }^{2,3,4,5}$
}

Published online: 26 June 2017

(C) Springer Science+Business Media, LLC 2017

Climate change is one of the biggest medium to long term risks to global development, both in developing and developed countries, and it is predicted to have severe consequences across such sectors as ecosystems, agriculture, industry, commerce, residences, and transportation. The threat of global climate change has originated largely from the greenhouse gas emissions of developed countries, who have been emitting far more per capita than developing countries over the last two centuries. Therefore, developed countries are obliged to take the lead in fighting climate change. However developing countries, especially emerging economies like China, also face tremendous pressure on the limited remaining space for atmospheric carbon dioxide. The processes of economic growth and social development must be rendered more resilient to climate change. Developed countries have to reduce their emissions, and developing countries need to find a low carbon path to rapid growth that is different from the energy intensive and carbon intensive path of early growth in developed countries. All must avoid needless local and regional environmental damage, capture innovative sources of sustainable development, and take advantage of emerging energy technologies and climate financing opportunities, so as to build up a low carbon economy and a climate resilient society.

Quantitative and model based analyses in energy economics systems and climate change economics systems are necessary to assess energy and climate policy decisions and further develop energy conservation and climate protection strategies. Modeling and decision making in energy and climate economics systems is a complex task, and the complexity of these systems derives from the nonlinear nature of society-economy-energy—environmenttechnique $\left(\mathrm{SE}^{3} \mathrm{~T}\right)$ interactions of the complex system. Given the global trend of environmental

Zhimin Huang

huang@ adelphi.edu

1 Robert B. Willumstad School of Business, Adelphi University, Garden City, NY, USA

2 Center for Energy and Environmental Policy Research and School of Management and Economics, Beijing Institute of Technology, Beijing, China

3 Beijing Key Lab of Energy Economics and Environmental Management, Beijing, China

4 Sustainable Development Research Institute for Economy and Society of Beijing, Beijing, China

5 Collaborative Innovation Center of Electric Vehicles in Beijing, Beijing, China 
awareness and low carbon development, energy policy and strategy design and assessment has become a nonlinear and dynamic problem. Not only do the socio-economic and technical concerns need to be included, but, furthermore, the constraints on environmental regulation and climate change mitigation and adoption have to be considered. The nonlinearity and dynamics of the complex energy and climate economics system are represented in the uncertainties of energy supply and demand, the prices of fossil fuels, non-fossil fuels, and carbon emission trading, the advantages and challenges of energy production and consumption related technologies, especially the new energy and clean energy technologies, the impact of energy combustion related carbon and pollutant emissions, and various regulatory regimes on energy conservation, energy efficiency, greenhouse gas emission reduction, energy security, and energy poverty.

Under such conditions, energy and climate change economics modeling and the applications of model-based simulation, evaluation, analysis, and design in energy and climate policy and strategy have seen tremendous growth during the last decade. Therefore, the primary aim of this special issue is to promote and disseminate the roles and applications of various modeling approaches to energy economics and climate change economics so as to improve the usefulness of energy and climate policy models in policy design and public decision making.

To accomplish a wider dissemination of the recent studies in energy economics and climate policy modelling, an open call for papers in the Annals of Operations Research (AOR) was announced. After a thorough and high-standard refereeing of more than one hundred submissions, 29 papers were finally accepted for publication. These papers cover a variety of research topics of interest in energy economics and climate policy modelling. They deal with problems in the areas of Integrated Assessment Modelling (IAM) for climate governance, carbon trading scheme and its influence on modelling, energy efficiency and ecological efficiency evaluation modelling, optimal decision making modelling under climate regulation, energy consuming and green consumer behavior modelling, and general energy management and environmental policy modelling.

With respect to IAM for climate governance, Z. Yang provides evidence of the likelihood of environmental coalitions and the number of coalition members. He points out that to identify the size of the core allocations is of vital importance for understanding the environmental coalition, since when holding the grand coalition voluntarily in an economy with detrimental externalities, the allocations should be in that core. In this paper, he constructs a cooperative game of stock externality provision and develops associated methods and algorithms for testing the shrinking core hypothesis based on the Regional Integrated Climate-Economy (RICE) model. His calculation results indicate that the size of the core shrinks as the number of regions increases in the RICE model.

M. Leimbach, A. Schultes, L. Baumstark, A. Giannousakis and G. Luderer present two solution algorithms of large-scale IAM of climate change: the well-known Negishi algorithm and a newly developed Nash algorithm. These algorithms are used to calculate the Paretooptimum and the competitive equilibrium, respectively, for the global model that includes trade in a number of goods as an interaction between regions. Their results show that in the absence of externalities, both algorithms deliver the same solution. In the presence of externalities between regions, the two solutions differ. This was demonstrated by the inclusion of global spillovers from learning-by-doing in the energy sector. The non-cooperative treatment of the spillover externality in the Nash algorithm leads to a delay in the expansion of renewable energy installations compared to the cooperative solution derived using the Negishi algorithm. 
With respect to a carbon trading scheme and its influence on modelling, M. A. Boutabba and S. Lardic provide an analysis of the competitiveness and carbon leakage from cement and steel industries under the European Union Emissions Trading Scheme (EU ETS). As the world's first large implementation of a carbon emissions trading system, the EU ETS would have adverse effects on industry sectoral competitiveness. This had become a major concern of policy makers and industry enterprises. In this paper, the authors examine whether and to what extent the cement and steel industries are exposed to carbon leakage with the consideration of structural breaks. Their rolling cointegration approach based estimation shows that carbon price positively affects the net imports of cement and steel sectors, which indicates that these two industries are affected by negligible carbon leakage and competitiveness losses. However, the steel sector is more affected than the cement sector.

Based on an oligopolistic partial equilibrium model with a detailed technological representation of the whole cement production process, E. Allevi, G. Oggioni, R. Riccardi, and M. Rocco investigate the role played by the transportation costs in cement production relocation and evaluate the effectiveness of carbon emissions allowance grandfathering and the effectiveness of the application of long-term power contracts in mitigating this phenomenon. The results of this paper show that the European and Italian cement markets were affected by the EU ETS and reacted by importing clinker from unregulated regions. Both allowance grandfathering and long-term power contracts only partially mitigate this relocation problem.

S. Yu, H. P. Weikard, X. Zhu and E. C. van Ierland develop a model to simulate the international carbon market in which the carbon emissions allowances are endogenously determined by each member of a carbon trade agreement but the constraint on the number of allowances per member is exogenously given. The STACO model, which is a numerically calibrated model with twelve world regions, is used to explore the incentive of each region to participate in this carbon market. Their results show that the stability and effectiveness of an international carbon market can be improved by imposing constraints on individual allowance choices compared to a carbon market without such constraints.

Y. J. Zhang and J. F. Hao develop a carbon emissions quota allocation model with both the equity and the efficiency principles for China's industry sectors. They construct a comprehensive index combined with the subjective, objective, and linear combination weighting methods to allocate carbon emission quotas among China's 39 industry sectors. The input oriented zero sum gains data envelopment analysis (DEA) model is utilized to examine the efficiency of the allocation solutions derived from their carbon emissions quota allocation model.

Y. Cai, Y. Lu, A. Stegman and D. Newth discuss the intensity based emissions reduction problem in which the reductions in emissions are specified with reference to some measure of economic output. The existing of intensity targets (e.g., the Copenhagen commitments of China and India) substantially increase the complexity of policy simulation and analysis, because a given emissions intensity target could be satisfied with a range of emissions and output combinations. The authors develop a simple iterative algorithm in this paper for an energy economic model to find a unique policy solution that achieves an emissions intensity target at minimum economic loss. They prove the mathematical properties of the algorithm and compare its numerical performance with other methods.

B. J. Tang, P. Q. Gong and C. Shen propose three hypotheses on the factors of carbon price volatility and apply ensemble empirical model decomposition and variance ratio to analyze the carbon price volatility of the EU ETS and clean development mechanisms (CDM). The results show that from the comparison of the two markets, not only in duration, but also in amplitude, the market mechanism and heterogeneity environment have a more significant impact on EU ETS than on CDM. 
J. Chevallier and S. Goutte propose an estimation methodology for Lévy-driven OrnsteinUhlenbeck processes. This estimation unfolds in two steps, with a least squares method for a subset of parameters in the first stage and a constrained maximum likelihood for the remaining diffusion and Lévy distribution parameters. They develop this estimation procedure to demonstrate that the class of mean-reverting Lévy jump processes provides a better fit of the electricity and carbon market prices.

This special issue includes eight papers on energy efficiency and ecological efficiency evaluation modelling. Most of them develop advanced non-parametric optimization models for efficiency measurement taking into consideration undesirable outputs (e.g., carbon emissions and air pollutant emissions). S. Lozano proposes a network DEA model that considers a production stage followed by a pollutant abatement stage, which is further utilized for separately assessing the technical and environmental efficiency of a network production system. In the model, the technical efficiency is calculated through a slacks based inefficiency measure, while the environmental efficiency is computed through adjusting pollutant emissions according to their environmental impacts and allowance costs. One key feature of this model is that it assumes all inputs, outputs, and intermediate measures that contain pollutants are not freely disposable, thus the materials balance principle (MBP) is utilized in the modelling.

B. Arabi, S. M. Doraisamy, A. Emrouznejad and A. Khoshroo also emphasize the introduction of MBP in industrial and agricultural performance measurement systems where the pollutant factors are taken into account. Their paper addresses an issue of eco-efficiency measurement adjusted for pollution, taking into account materials flow conditions and the MBP requirements, so as to provide a "real" measure of performance that can guide policy making. The model proposed in this paper integrates a slacks based measure that enhances the Malmquist-Luenberger Index, and a material balance condition that reflects the conservation of matter. Their model is demonstrated to measure the productivity and eco-efficiency trends of power plants.

P. Zhou, F. Wu and D. Q. Zhou propose a total factor energy efficiency (TFEE) measurement with the congestion effect taken into account. They provide a definition of congested production technology and, based on this, several DEA models are developed to construct the TFEE measurement with congestion. In addition, the models for quantifying energy inefficiency caused by congestion effect are developed in this paper. The authors applied the TFEE measurement to estimate the energy efficiency of China's industry sectors and found that this measurement can yield useful insights on suggesting proper ways to achieve energy efficiency promotion.

Three papers in this special issue focus on energy and environmental efficiency evaluation in China. J. Wu, B. Xiong, Q. An, J. Sun and H. Wu develop a two-stage DEA model for evaluating the TFEE of China's industry. The industrial system was divided into two sub stages, an energy utilization stage and a pollution treatment stage, for accurately evaluating the TFEE of the first stage and the overall efficiency of the whole system. L. Zhao, Y. Zha, K. Wei and L. Liang develop a target-based DEA method for energy saving and carbon emissions reduction, which is utilized to design the step-by-step mechanisms of energy saving and carbon emissions reduction in China. Y. Bian, K. Lv and A. Yu develop an interval slacks based measure approach for evaluating energy efficiency and carbon emissions efficiency in the presence of recovery energy and apply it to regional energy efficiency measures of China.

W. D. Cook, J. Du and J. Zhu use the DEA model to assess ecological efficiency where air pollutant emissions are used as undesirable outputs. The weight restrictions are incorporated in the modelling so as to reflect a user's preference over the performance metrics and to refine the DEA results. Since the introduction of weight restrictions voids unit invariance in DEA, this paper develops a modified DEA model for ecological efficiency assessment which 
is units-invariant when weight restrictions are imposed. Y. Chen, W. D. Cook, J. Du, H. Hu and J. Zhu discuss the modelling of bounded and discrete data as well as the Likert scales in DEA. They propose an updated DEA model for dealing with bounded and discrete data which addresses the issue of constraining DEA projections to fall within imposed bounds. The proposed DEA models are used to evaluate the energy efficiency of 29 provinces in China.

The fourth major topic of interest in this special issue is optimal decision making modelling under climate regulation. In B. Hampf and K. L. Rødseth's paper, on optimal profits of coal fired power plants in U.S. under environmental regulations, the economic effects of implementing the Environmental Protection Agency's (EPA's) newly proposed carbon regulations on 144 electricity generating units of U.S. coal fired power plants are analyzed through utilizing a nonparametric method. In addition, this paper develops an approach for evaluating the economic gains from averaging emission intensities among these electricity generating units, compared to implementing unit-specific performance standards. The results show that, compared with the uniform standards, the implementation of flexible standards leads to 2.7 billion dollars larger profits. However, there also exists a tradeoff between environmental gains and economic gains. The results indicated that the aggregate carbon emissions were higher with flexible standards than with uniform standards.

B. Chen, E. Klampfl, M. Strumolo, Y. Fu, X. Chao and M. A. Tamor's paper focusses on the optimal investment strategies for light duty vehicle and electricity generation sectors with carbon constrains. They point out that meeting the carbon emission targets for both automakers and electricity generators will require strategies for technology and capacity planning that are both cost efficient and environmentally conscious. In order to estimate the advantage of cooperation of light duty vehicles (plug-in hybrid and electric vehicles) and electricity generation sectors in meeting the carbon emission targets and to enhance the policies for encouraging their cooperation, a mathematical model for tradeoff analysis between these sectors if they work independently with separate emission constraints or together with a joint constraint is proposed in this paper. One of the key findings of this model was that although the potential savings are just $3 \%$ of the discounted total societal cost incurred in both sectors, cooperation can significantly reduce the incremental cost by $50 \%$ for carbon emission reduction. Another interesting result was that if the natural gas price keeps at a low level, to build natural gas generators will be more favorable than to build renewable generation capacity in the short run.

S. Yu, S. Zhang, L. Agbemabiese and F. Zhang propose a weighted goal programming model for optimizing crude oil production in the middle and later periods of oil field development. The oil yield optimization problem is divided into two stages in this model. In the first stage, the total planned crude oil yield is optimized by four sub-yields; and each sub-yield is then optimally allocated among different oil extract plants in the second stage. This model helps to allocate resources among various extract oil plants and to reduce production costs of planned oil yields.

The increased risks of climate change and energy price fluctuations make farmers' adaptations to them more and more important, since farmers are exposed to substantial weather and market related risks. Managing soil natural capital was proven to generate the double dividend that both increases farm profit and reduces the above-mentioned risk. R. G. Cong, M. Termansen and M. V. Brady explore whether the management of soil natural capital provides a third dividend of reducing downside risk. They propose a dynamic stochastic portfolio model to optimize the stock of soil natural capital and, based on this, they find that the management not only improves farm profit while reducing risk but it also reduces the downside risk. 
M. Chesney, P. Lasserre and B. Troja explore the optimal investment choices in global warming mitigation through introducing a tipping point in a real options model.

Four papers treat energy consuming and green consumer behavior modelling. G. Li, W. Liu, Z. Wang and M. Liu provide an empirical examination of the features of energy related situational factors, individual and household energy consumption behavioral norms, and energy saving behavioral intentions of residents in Beijing. The results of this paper show that situational factors most significantly and effectively influence the residents to assume energy saving behaviors.

M. Lu and Z. Wang discuss the rebound effects of residential electricity use in urban China. Applying 30 sets of provincial panel data and national input-output data for China in 2007, this paper builds a co-integrating equation, a panel error correction model, and an eight-sector energy input-output model. The evaluation results indicate that in the long run the direct plus indirect partial rebound effect is 0.79 , and in the short run it is 0.78 . Therefore, a major part of the expected electricity reduction in China's urban residential energy consumption arising from efficiency improvement has been offset.

G. Bi, M. Jin, L. Ling and F. Yang present a study on a subsidy policy from the government to motivate enterprises' adoptions of green technology for emissions reduction when consumers are environmentally discerning. They build a model that considers two profitmaximizing firms selling two products in a price and pollution sensitive market, and propose two cases. In one the government has a limited budget and can choose only one firm at most to provide subsidy; and in the second the government has a sufficient budget and can choose both firms to provide a subsidy. Based on this model, the authors discuss which firms should be selected in each case and in which situation the firm has incentive to invest in the green technology.

In the fourth paper, S. Du, L. Hu and L. Wang expand the environment view to supply chain operations. This paper develops a Stackelberg-like model for game-theoretically analyzing the decentralized decisions of the manufacturer and retailer where low-carbon effort is taken into consideration by both sides. Several managerial insights on low-carbon factors are proposed.

The remaining papers of this special issue are grouped into the topic of general energy management and environmental policy modelling. F. Fathurrahman, B. Kat and U. Soyta? propose a social accounting matrix based analysis for simulating Indonesia's fuel subsidy reform. This paper estimates the impacts of fuel subsidies from the economic, social, and environmental perspectives, and proposes policy options for a subsidy reform in Indonesia. The results show that reallocation of a fuel subsidy to other sectors might promote the overall economic development but compromises environmental aspects. However, to directly reallocate the subsidy to the low income households might slow down the overall economic development but results in a positive effect for social welfare.

H. Cao, T. Li, S. Li and T. Fan propose an integrated emergency response model for toxic gas release accidents in the energy and chemical industry, which consists of a toxic gas dispersion model, a dynamic evaluation model for accident consequences, and an evacuation route selection model. The first sub-model predicts the distribution of toxic gas concentration when a toxic gas release accident happens; the second sub-model estimates the consequences the accident; while the third sub-model provides the safest evacuation route for evacuees. Their integrated model is verified utilizing an ammonia gas release accident in an energy and chemical enterprise.

Finally, based on a variety-expanding endogenous technological change model, W. Jin and Z. X. Zhang investigate the economic mechanism of the slow pace of energy technological progress. The results show that in the market equilibrium, the growth rate of energy technol- 
ogy is always lower than that of IT. This outcome stems from both the market fundamentals, where the homogeneity of end use energy goods is less likely to harness the pecuniary externality embedded in the love-for-variety household, and the technology fundamentals, where capital intensiveness of energy technology assets inhibits the non-pecuniary technological externality associated with knowledge spillovers. In addition, the social planner allocation can raise the rate of technological progress in both energy and IT sectors, but still fails to achieve an outcome in which technological progress in the energy sector can catch up with the IT sector.

We are very glad to have been guest editors for the special issue on energy economics and climate policy modeling. We do hope the issue will be of interest to academic and industrial communities, as well as to government policy makers for helping them to understand the complexity of energy and climate economics systems and the necessity of quantitative modelling of these systems.

In closing, we thank the anonymous referees for their diligent efforts and constructive feedback during the entire review process. We gratefully acknowledge Professor Endre Boros, Editor-in-Chief of AOR, Katie D'Agosta, Publications Manager of AOR, and Anandhi Sankar, Journals Editorial Office Assistant of AOR, for supporting the special issue and offering their expert guidance and efforts.

Acknowledgements We acknowledge the financial support from the National Natural Science Foundation of China under Grant Nos. 71322306, 71471018, 71521002, and 71642004; the Social Science Foundation of Beijing under Grant No. 16JDGLB013; the Joint Development Program of Beijing Municipal Commission of Education; and the National Key R\&D Program under Grant No. 2016 YFA0602603. 